\title{
Mudança de hábitos e atitudes em sobreviventes de infarto agudo do miocárdio e angioplastia primária
}

\author{
Adriana Martins Gallo ${ }^{1}$ Ruy Laurenti ${ }^{2}$
}

\begin{abstract}
RESUMO
O objetivo do estudo foi identificar mudanças de hábitos e atitudes em sobreviventes de infarto agudo do miocárdio após intervenção coronária percutânea (ICP). Trata-se de pesquisa descritiva, com abordagem quantitativa dos dados referentes à aplicação de um questionário estruturado à pacientes submetidos à ICP, durante internação hospitalar após infarto agudo do miocárdio (IAM). 0 questionário foi composto por cinco etapas relacionadas aos fatores de risco (FR) e comportamenos para as doenças cardiovasculares (DCV) durante dois períodos da vida -antes e após a ocorrência do IAM. Os momentos foram comparados entre si por meio do teste "t" de Student adotando-se o nível de $5 \%$ de significância. 0 estudo demonstrou que houve mudanças após o infarto em hábitos e atitudes relacionados à alimentação, exercícios físicos, controle de níveis glicêmico e lipídeos e cessação do tabagismo, além de maior preocupação e cuidados em relação à saúde.
\end{abstract}

Descritores: Angioplastia; Sobrevivência; Infarto do Miocárdio; Fatores de Risco; Doenças Cardiovasculares

\section{Changing habits and attitudes in survivors of acute myocardial infarction and primary angioplasty}

\begin{abstract}
The aim of the study was to identify changes in habits and attitudes in survivors of acute myocardial infarction after percutaneous coronary intervention $(\mathrm{PCl})$. This is a descriptive study with a quantitative approach to the data relating to the application of a structured patients undergoing PCl during hospitalization after acute myocardial infarction (AMI) questionnaire. The questionnaire was composed of five steps related to risk factors (RFs) for cardiovascular disease (CVD) during two periods of life before and after the occurrence of AMI. Times were compared using the " $\mathrm{t}$ " test adopting the $5 \%$ level of significance test. The study showed that there were changes after infarction in habits and attitudes related to diet, exercise, glycemic control and lipid levels and smoking cessation, as well as greater concern and care for their health.
\end{abstract}

Descriptors: Angioplasty; Survival; Myocardial Infarction; Risk Factors; Cardiovascular Diseases.

\footnotetext{
${ }^{1}$ Mestre em Saúde coletiva pela Universidade do Sagrado Coração (USC), Bauru, SP, Brasil

2 Doutor em Cardiologia pela Universidade de São Paulo (USP), São Paulo, SP, Brasil.
} 


\section{Introdução}

As doenças cardiovasculares (DCV) representam a primeira causa de morte no Brasil, e aproximadamente $80 \%$ estão associados a fatores de risco (FR), que inclui características constitucionais - sexo, idade, raça e genética; características comportamentais - tabagismo, alimentação e atividade física; patologias ou distúrbios metabólicos - hipertensão arterial, obesidade, hiperlipidemia, diabetes mellitus; e características socioeconômicas - renda, ocupação, escolaridade e classe social'.

Dentre as DCV, os distúrbios isquêmicos do coração destacam-se como as principais causas de óbito, em especial o Infarto Agudo do Miocárdio (IAM). A presença de FR, principalmente quando associados entre si, pode contribuir para 0 aumento do número de indivíduos acometidos por complicações decorrentes de um IAM².

Depois de ocorrido um IAM e realizado o atendimento hospitalar imediato, provavelmente, em curto período, 0 paciente recebe alta hospitalar e deste então, a adoção de mudanças de hábitos e atitudes irão amenizar o risco de desenvolvimento de um novo IAM, por meio da prevenção secundária ${ }^{3,4}$.

Em alguns casos, o autocuidado proposto para a prevenção secundária das DCV, conduz o indivíduo a sair de sua zona de conforto, adotando mudanças comportamentais radicais, dentre elas a redução de peso, por meio de dieta hipocalórica, a diminuição da ingestão de sal, a adoção de atividade física regular, e a cessação do tabagismo. Essas atitudes são comprovadamente eficazes para a diminuição do risco cardiovascular ${ }^{5}$.

Vale salientar que, pacientes acometidos por IAM, geralmente, não percebem a gravidade da sua doença e a necessidade de cuidados posteriores, em decorrência do curto período de hospitalização, embora tenham sido submetidos à angioplastia primária. Nesse sentido, faz-se necessária uma abordagem multifatorial, ou seja, explicando aos pacientes quais são os FR mais importantes que estavam presentes em suas vidas antes de acontecer o IAM e são as reais necessidades de adaptações benéficas de comportamento a partir daquele momento.

Muitos dos pacientes infartados, mesmo internados por um quadro agudo, quando passam a entender sua classificação como grave, possuem condições de perceber que o impacto da mudança, a partir de então, de seus hábitos e atitudes serão determinantes na redução e controle dos FR que podem agravar a doença acometida, no caso o IAM. Desta forma, a prevenção secundária é o caminho mais coerente, visto que, para evitar uma recorrência da doença, é preciso que a adesão às alterações no estilo de vida, incluindo também, o tratamento farmacológico, se torne a prioridade na vida dos pacientes ${ }^{6}$.

Considerando a importância da prevenção secundária do IAM, o presente estudo teve como objetivo identificar mudanças de hábitos e atitudes em sobreviventes de IAM após ICP.

\section{Material e métodos}

Trata-se de pesquisa descritiva, com abordagem quantitativa dos dados referentes à aplicação de um questionário estruturado à pacientes submetidos à ICP, durante internação hospitalar após IAM, residentes em dois municípios da região Norte do Paraná. O primeiro é o município sede do hospital onde os pacientes foram internados e o segundo é o município sede da regional de saúde ao qual o primeiro município pertence. 0 referido hospital atua em média e alta complexidade, com atendimento vinculado ao Sistema Único de Saúde.

Enquanto internados, os pacientes que possuíam diagnóstico médico de IAM foram abordados por uma enfermeira, que realizou orientações quanto aos métodos de prevenção secundária, readaptação da rotina a partir da alta hospitalar, e mudanças de hábitos e atitudes que contribuíssem para a inibição dos FR potencialmente modificáveis.

Os participantes do estudo foram acessados a partir do levantamento das internações do período de janeiro de 2007 a dezembro de 2008. Neste período, foram internadas, aos cuidados da clínica cardiológica, 5.136 pessoas, destas, 2.139 passaram por intervenção cirúrgica, sendo realizado cateterismo cardíaco e/ou angioplastia e/ou cirurgia cardiovascular. O total de indivíduos com diagnóstico médico de IAM foi de 213. Para a seleção da população do estudo, 0 indivíduo deveria ter residência fixa em um dos dois municípios escolhidos, apresentar diagnóstico de IAM na internação, terem sido submetidos à ICP primária, e ter recebido alta hospitalar por melhora do quadro clínico a mais de 12 meses. A partir dos critérios de inclusão foram selecionados 52 pacientes, destes, seis foram excluídos por evoluírem para óbito e outros seis por não terem sido localizados. Portanto, a população do estudo foi composta de 40 pacientes. 
As visitas foram realizadas nos meses de janeiro a março de 2010 , sendo a abordagem inicial ao paciente por meio de contato telefônico, para agendamento do encontro domiciliar e aplicação do questionário estruturado. Para os casos em que o contato telefônico não se efetivou, foi realizado visita sem agendamento prévio e a abordagem inicial foi 0 esclarecimento das etapas da pesquisa e aplicação do questionário.

0 questionário estruturado foi elaborado pelos pesquisadores, tomando como base dados da literatura ${ }^{3,7-9}$. Composto por cinco etapas relacionadas aos FR para as DCV durante dois períodos da vida - antes e após a ocorrência do IAM. A primeira etapa continha questões referentes ao perfil sócio demográfico; a segunda versou sobre condições de saúde e rotinas diárias dos pacientes; a terceira relacionada a doenças pré-existentes, utilização de medicamentos, e medidas de controle; a quarta referente ao índice de massa corporal (IMC) e peso corporal no momento da entrevista e no último ano; e, a quinta e última etapa, relacionada ao hábito de fumar, atividades sexuais e estresse.

Após a realização de um piloto do questionário com 10 pacientes, foram realizadas reformulações quanto à interpretação de perguntas e respostas, para facilitar a compreensão do entrevistado. As entrevistas do teste piloto foram incluídas no plano amostral.

As variáveis de caracterização sociodemográfica foram representadas por meio de frequência absoluta e relativa. Os momentos foram comparados entre si por meio do teste "t" de Student adotando-se o nível de 5\% de significância.

O teste "t" conduzido para comparar as amostras pareadas, ou seja, os mesmos sujeitos em dois momentos distintos, sendo o primeiro momento aquele que se referia o período antecedente ao IAM e o segundo momento há pelo menos um ano após a alta hospitalar após ICP primária.

A presente pesquisa foi aprovada pelo Comitê de Ética e Pesquisa da Universidade Sagrado Coração, sob o parecer $n^{\circ} 102 / 09$. Os dados foram coletados somente após o entrevistado aceitar a participar da pesquisa e assinar o Termo de Consentimento Livre e Esclarecido (TCLE) em duas vias, obedecendo aos princípios éticos estabelecidos pela Resolução do Conselho Nacional de Saúde $n^{\circ} 466 / 12^{10}$.

\section{Resultados}

A maioria dos pacientes eram homens (60\%), a faixa etária predominante foi entre 51 e 60 anos (37,5\%), 90\% viviam com companheiro (a), e 35,0\% possuíam até quatro anos de estudo Tabela 1.

Os resultados apontam para um aumento do número de refeições durante o dia, bem como da ingesta de frutas entre os pacientes - antes do IAM apenas 20,0\% faziam quatro refeições por dia, e após aumentou para 55,0\%; a ingesta de frutas por no mínimo duas vezes por semana, aumentou de $12,5 \%$ para $35,0 \%$. A ingestão de gordura e fritura teve redução significativa após o IAM, de 27,5\% para 7,5\%. A utilização de saleiro à mesa diminuiu consideravelmente Tabela 2.

Quanto à realização de atividades físicas os resultados apontaram que após o IAM o percentual de pacientes que não realizavam atividades físicas caiu de $52,5 \%$ para $32,5 \%$. Após o IAM, o percentual de pacientes que realizavam atividades físicas duas vezes por semana, aumentou de 5\% para 30\%, associando-se ao aumento do percentual de prescrição pelo profissional de saúde (de 15\% para 60\%).

A Tabela 3 aponta que tanto para hipertensão, quanto para diabetes não houve diferenças significativas antes e depois do IAM, porém as pessoas passaram a adotar condutas medicamentosas para controle da hipertensão e ainda, fazer controle da glicemia com uso de insulina após o IAM. Também, os pacientes passaram a verificar mais vezes a pressão arterial além de fazer o controle da glicemia capilar.

Sobre o nível glicêmico, 87,5\% dos entrevistados referiram nunca ter feito o teste de glicemia capilar antes do IAM, sendo que no período após o IAM, 32,5\% disseram realizar tal teste pelo menos uma vez ao mês.

Os resultados demonstraram que a dislipidemia permaneceu sem diferenças significantes nos dois momentos, mas, notou-se significância estatística na adesão ao tratamento medicamentoso e também que passaram a evitar a ingesta de gorduras e frituras após o IAM ( $25 \%$ no período anterior ao IAM para $85 \%$ após) (Tabela 3 ).

A frequência de indivíduos com excesso de peso após o IAM foi de $55 \%$, e com obesidade grau I representou $20 \%$ da população. Em relação ao último ano, os entrevistados autorreferiram seu peso, sendo que $40 \%$ dos entrevistados disseram ter diminuição do peso, enquanto $37,5 \%$ disseram manter o peso anterior a este período (Tabela 4).

O tabagismo antes do IAM foi relatado por $45 \%$ dos entrevistados, sendo que este percentual reduziu para $7,5 \%$. Destaca-se que $15 \%$ pararam de fumar antes do episódio agudo do infarto. (Tabela 5) 
Em relação à vida sexual (Tabela 5), antes do IAM, 90\% eram sexualmente ativos e a maior parte (37,5\%) mantinha de seis a dez relações sexuais por mês. Comparando-se a satisfação da relação sexual, observou-se significância estatística sobre a qualidade da relação, pois antes do IAM a maioria das pessoas eram satisfeitas (85\%) e após o IAM, as pessoas satisfeitas representavam $17,5 \%$.

Os entrevistados relataram que possuíam atitudes positivas em relação à própria vida antes e depois do IAM (45 \% e $87,5 \%$ respectivamente).

\section{Discussão}

No presente estudo, identificou-se que as pessoas saem do momento agudo da doença cada vez mais conscientes que devem mudar seus hábitos e atitudes, da mesma forma que resultados apontados por outras pesquisas ${ }^{2,11}$ em que ficou evidenciado que as mudanças aliadas a hábitos e posturas inadequadas à saúde, como inatividade física e tabagismo, contribuem fortemente para expor ainda mais os indivíduos às doenças, especialmente à recorrência das DCV.

Ao receber alta hospitalar após o tratamento para o IAM, o paciente teme a recorrência da doença. Este fato gera uma sensação de impotência, prostração e a incerteza de recuperação ${ }^{6,12}$. Identificou-se no período que antecede à alta hospitalar a ansiedade em querer mudar e modificar os FR.

Os pacientes após o IAM, passam a antecipar a vida após a alta6, de um lado querendo viver intensamente, com receio de morrer de forma súbita e por outro, tendo que limitar-se às novas regras sugeridas pelos profissionais da saúde que passam a acompanhá-lo, e por meio de programas de educação em saúde, Ihes repassam as informações e sugestões de algumas limitações a serem adotadas. Estas variam muito de acordo com o estilo de vida que o paciente estava acostumado no período anterior ao IAM.

Neste sentido vale salientar a importância da orientação sobre a prevenção secundária ainda no período de internação, mesmo que qualquer ação eficaz para a mudança de atitude, somente poderá ocorrer se a pessoa estiver motivada e contar com suportes imprescindíveis de estrutura econômica e social ${ }^{3}$.

Assim como em um estudo transversal em Florianópolis ${ }^{12}$, este estudo mostrou que o consumo saudável de alimentos que inclui frutas, verduras e legumes não estão adequados ao ideal e que menos de um quarto da população adulta entrevistada por telefone atingiu a recomendação de consumo adequado destes ítens. Salienta-se que promover o aumento da ingestão destes itens na população é uma questão de saúde pública em vários países, uma vez que este hábito é benéfico no na prevenção da obesidade e do excesso de peso, diabetes e, consequentemente, das DCV.

Deste problema podem surgir algumas considerações importantes, dentre elas, pode-se citar os agravos à saúde, uma vez que a obesidade, a inatividade física, a hipertensão arterial e o tabagismo são os principais fatores de risco para as DCV ${ }^{13}$.

A realização de atividade física superior a 30 minutos, seja no lazer, no trabalho ou no deslocamento corriqueiro diário, mostrou ser estatisticamente significante na comparação entre os momentos, bem como a realização destas atividades por prescrição médica, sendo que a caminhada é a preferência dos infartados.

Algumas doenças como as cardiovasculares, diabetes e alguns tipos de câncer são menos propensas a se desenvolverem quando o indivíduo pratica regularmente atividade física. Esta prática produz diferentes benefícios proporcionados à saúde além de combater eficazmente o sedentarismo, e ainda, estimula a retomada de alguns hábitos de vida ${ }^{12,14,15}$. Sendo assim, tornou-se um recurso a ser utilizado também na prevenção secundária e vêm ganhando relevância nas políticas públicas de promoção à saúde, principalmente quando há treinamento supervisionado ${ }^{16}$.

Sobre a prevalência de hipertensão, diabetes e dislipidemia, os resultados corroboraram com outro estudo ${ }^{17}$ que não apresentaram diferenças estatísticas antes e após o IAM.

Destaca-se que nesta investigação a adesão à prevenção secundária relacionada aos hábitos e atitudes foi positiva pela maior parte dos infartados, contradizendo outros apontamentos ${ }^{18-20}$.

Identificou-se também, que após o IAM houve uma redução da utilização de saleiro à mesa, o que sugere que a indicação médica de redução na ingestão de sal está sendo cumprida, assim como em outro estudo ${ }^{19}$.

O hábito de fumar, diminuiu após o IAM, sendo estatisticamente significante. Os mesmos resultados foram encontrados também em outros estudos e destaca-se similaridade com o trabalho publicado por Scherr ${ }^{5}$ que estudou a avaliação do impacto de diminuição dos FR após palestras educativas, onde o tabagismo era prevalecente em $60 \%$ dos entrevistados 
e, após ICP, 9\% cessaram o hábito e 32\% diminuíram. Da mesma forma, Vega e Martinez ${ }^{17}$ demonstraram que pacientes que fumavam no momento do IAM, após orientação sobre a cessação do fumo, pararam ou diminuíram a quantidade de cigarros por dia.

Foram relatados resultados semelhantes ${ }^{21}$ no que concerne à dificuldade encontrada para lidar com as potencialidades humanas, demonstrando a presença de estresse no cotidiano, porém sem diferença estatisticamente significante. Ressalta-se também que analogamente ao município de São $\mathrm{Paulo}^{3}$, os pacientes viam-se com atitudes positivas perante a saúde e que o estresse não atrapalhava as atividades corriqueiras.

No que diz respeito a um novo IAM, os resultados demonstraram que os pacientes o temem, assim como em outros estudos $^{6,21,22}$ e sendo assim, começam a pensar a vida em busca de caminhos para sobreviver; julgando racionalmente o cotidiano, passando a aceitar padrões de comportamento a serem adotados. E, entretanto, transitam para a vida cotidiana com a insegurança da "cura", a imprevisibilidade do que pode acontecer, o temor do retorno da doença.

Referindo-se à adesão à prevenção secundária, ficou demonstrado que houve diferença estatística significante nos momentos avaliados para diabetes, hipertensão, hipercolesterolemia e obesidade após o IAM, diferentemente dos resultados obtidos por Vega e Martinez ${ }^{17}$ que apontaram que os pacientes portadores destas doenças seguiam um controle inadequado após o IAM.

Neste estudo não houve diferença significativa no que se refere à quantidade de relações sexuais por mês antes e depois do IAM, fato este que contradiz resultados de estudos de mesmo cunho ${ }^{23}$ que indicam que os distúrbios sexuais após o IAM, causam redução da frequência das atividades entre $40 \%$ a $70 \%$ dos casos. Neste observou-se insatisfação sexual, com significância estatística, podendo inferir em piora da qualidade das atividades sexuais, corroborando com outro estudo ${ }^{24}$ que indica impacto desfavorável para a variável satisfação sexual.

Demonstrou-se que, mesmo havendo mudanças positivas em relação aos hábitos e atitudes dos pacientes, a efetivação da prevenção secundária não se deu por completo. Assim também está exposto nacionalmente ${ }^{5,18,24,25}$, que o controle dos FR associados às terapias medicamentosas geram um impacto favorável principalmente àqueles que já infartaram anteriormente, porém não se sabe ao certo o motivo pelos quais os programas de prevenção secundária e suas recomendações e estratégias de manejo em prática clínica ainda não se efetivam.

O que se demontra é que o indivíduo usa de forma desregrada as informações repassadas nos programas preventivos, e muitas vezes este fato está ligado aos valores culturais que envolvem o ser humano 6,12,14,15.

A prevenção secundária para reabilitação cardíaca não consiste apenas em diminuir o peso, modificar a alimentação e praticar exercícios, mas também, envolve a participação de equipe multiprofissional que envolva o paciente em um programa de prevenção secundária capaz de reduzir todos os FR modificáveis para DCV.

\section{Considerações finais}

Verificou-se que após a orientação recebida houve mudanças de alguns hábitos e atitudes relacionados à alimentação, ingestão de sal, atividades físicas orientadas por profissionais, controle de hipertensão, níveis glicêmicos, lipídicos e cessação ao tabagismo. Ressalta-se a não modificação de alguns comportamentos e hábitos como utilização de açúcar, estresse cotidiano, quantidade mensal de relações sexuais, mesmo que o contentamento sexual atual tenha sido relatado como insatisfatório quando comparado ao período anterior ao IAM.

Neste estudo foi possível identificar que a maioria dos pacientes aderiu às recomendações do programa de prevenção secundária proposto no período que antecedia sua alta hospitalar, sugerindo que há uma preocupação elevada por parte dos pacientes em relação às mudanças de hábitos que diminuem FR potencialmente modificáveis.

Embora os resultados apresentados refiram-se a apenas dois municípios da mesma região, o presente estudo é importante pela contribuição aos dados nacionais, possibilitando a replicação do estudo a outras realidades. Destacase também a importância deste resultado como contribuição para a melhoria da atuação dos profissionais da saúde em relação à prevenção secundária, especialmente aqueles que lidam diretamente com o paciente durante a internação e fase aguda da doença. 


\section{Referências bibliográficas}

1. Spinel LF, Puschel VAA. Perfil de estilo de vida de pessoas com doença cardiovascular. Rev Gaucha Enferm. 2007; 28(4):534-41.

2. Pinheiro RHO, Vieira MCU, Pereira EM, Barbosa MEM. Fatores de risco para infarto agudo do miocárdio em pacientes idosos cadastrados no programa Hiperdia. Cogitare Enferm. 2013; 18(1):78-83.

3. Borges, ES. Insuficiência Coronariana. Manual de Educação Continuada da Sociedade Brasileira de Cardiologia. SBC [Internet]. 2006 [cited 2009 mar]. Disponível em: http://educacao.cardiol.br/manualc/PDF/i_insuficiencia_coronariana_ novo.pdf

4. Santos Filho EG; Abdo GL; Orel M; Gun C. Prevenção da reestenose pós-angioplastia coronária: mito ou realidade?. Rev Med. 2002; 81(1/4): 31-41.

5. Scherr C, Cunha AB, Magalhães CK, Abitibol RA, Barros M, Cordovil I. Intervenção nos hábitos de vida em instituição pública. Arq Bras Cardiol. 2010; 94(6):730-37.

6. Mussi FC, Koizumi MS, Angelo M, Lima MS. Perda da espontaneidade da ação: o desconforto de homens que sofreram infarto agudo do miocárdio. Rev Esc Enferm USP. 2002;36(2):115-24.

7. Word Health Organization. (WHO - Technical Repor Series) Geneva: 2002.Disponível em: <http://www.who.int/ whr/2002/en/>. Acesso em: 04 jun 2010.

8. Le May MR, So DY, Dionne R, et al. A citywide protocol for primary PCI in ST-segment elevation myocardial infarction. N Engl J Med. 2008;358:231-40.

9. Avezum A, Carvalho ACC, Mansur AP, Timerman A, Guimarães AC, Bozza AEZ. et al . III Diretriz sobre tratamento do infarto agudo do miocárdio. Arq Bras Cardiol. 2004; 83(4):1-86.

10. Conselho Nacional de Saúde. Resolução n 466 de 12 dezembro de 2012. [Internet]. Disponível em http://conselho. saude.gov.br/resolucoes/2012/Reso466.pdf Acesso em: 06 jun 2014.

11. Laurenti R, Buchalla, CM. Myths About Cardiovascular Diseases. Arq Bras Cardiol. 2001;76(2):105-10.

12. Campos VC, Bastos JL, Gauche H, Boing AF, Assis MAA. Fatores associados ao consumo adequado de frutas, legumes e verduras em adultos de Florianópolis. Rev Bras Epidemiol. 2010;13(2):352-62.

13. Santos VEP, Cruz NM, Moura LA, Moura JG, Matos KKC. O estado nutricional e o comportamento alimentar de um grupo de mulheres na estrategia de saúde da família. Rev Enferm UFSM. 2012;2(2):394:400.

14. Zanchetta LM, Barros MBA, César CLG, Carandina L, Goldbaum M, Alves MCGP. Inatividade física e fatores associados em adultos, São Paulo, Brasil. Rev Bras Epidemiol. 2010;13(3):387-99.

15. Formiga ASC, Dias, MR, Saldanha, AAW. Aspectos psicossociais da prevenção do infarto: construção e validação de um instrumento de medida. Psico-USF. 2005;10(1): 31-40.

16. Kalka D, Sobieszczanska M, Pilecki W, Adamus J.Complex cardiac rehabilitation in a strategy of secondary prevention of cardiovascular disease. Pol Merkur Lekarski. 2009; 27(157):30-35.

17. Vega G, Martinez S. Perfil del paciente con seguimiento y control inadecuados de los factores de riesgo cardiovascular después de presentar un infarto agudo de miocardio. Aten Primária. 2008;40(10):497-503

18. Oliveira KCS. Fatores de Risco em pacientes com infarto agudo do miocárdio em um hospital privado de Ribeirão Preto-SP [dissertação]. Ribeirão Preto (SP): Universidade de São Paulo; 2004.

19. Rosário TM, Scala LCN, França GVA, Pereira MR, Jardim PCBV. Fatores associados à hipertensão arterial sistêmica em Nobres-MT. Rev Bras Epidemiol. 2009;12(2):248-57.

20. Yusuf S, Hawken S, Ònpuu S, Dans T,Avezum A,Lanas F, et al. Effect of potentially modifiable risk factors associated with myocardial infarction in 52 countries (the INTERHEART study): case-control study. Lancet. 2004;364:937-52.

21. Santos FLMM, Araújo TL. Vivendo infarto: os significados da doença segundo a perspectiva do paciente. Rev. LatinoAm. Enfermagem. 2003;11(6): 742-48.

22. Oberg EB, FitzpatrickAL, Lafferty WE, LoGerfo JP. Secondary prevention of myocardial infarction with nonpharmacologic strategies in a Medicaid cohort. Prev Chronic Dis. 2009;6(2):1-9.

23. Vacanti LJ, Caramelli B. Idade e distúrbios psicológicos: variáveis associadas à disfunção sexual no período pósinfarto. Arq Bras Cardiol. 2005;85(2):110-14.

24. Jardim PCBV, Gondim MRP, Monego ET, Moreira HG, Vitorino PVO, Souza WKSB et al . Hipertensão arterial e alguns fatores de risco em uma capital brasileira. Arq Bras Cardiol. 2007; 88(4):452-57. 
25. Sturmer G, Dias-da-Costa JS, Olinto MTA, Menezes AMB, Gigante DP, Macedo S. O manejo não medicamentoso da hipertensão arterial sistêmica no Sul do Brasil. Cad Saúde Pública. 2006; 22(8):1727-37.

\section{Adriana Martins Gallo}

Endereço para correspondência - Rua João XXIII, Praça Horace Wells, 600. Bairro Judith, CEP 86060-370, Londrina, PR, Brasil.

E-mail: adriana.gallo@ifpr.edu.br

Lattes: http://lattes.cnpq.br/7025711277523434

Ruy Laurenti - laurenti@usp.br

Enviado em 26 de março de 2014.

Publicado em 07 de julho de 2014. 
